\title{
Application of a new variational functional for electron-molecule collisions: an extension of the Schwinger variational principle
}

\author{
Lee Mu-Tao†, Kazuo Takatsuka and Vincent McKoy \\ A A Noyes Laboratory of Chemical Physics $\ddagger$, California Institute of Technology, Pasadena, \\ California 91125 , USA
}

Received 18 June 1981

\begin{abstract}
We discuss a variational functional for scattering theory which has been recently proposed by Takatsuka and McKoy. It is shown that this functional can provide results with a purely discrete set of functions which are approximately equivalent to those obtained by Lucchese et al from the first iteration of the iterative Schwinger method. Applications to the scattering of electrons by systems including $\mathrm{CO}^{+}$and $\mathrm{LiH}$ illustrate this relationship and other features of the method.
\end{abstract}

\section{Introduction}

Recently we have shown that the Schwinger variational principle for scattering theory (Blatt and Jackson 1949, Kato 1951) is an effective approach to the electron-molecule collision problem (Watson et al 1980, Lucchese and McKoy 1979, 1980). Although one can use trial scattering wavefunctions consisting entirely of discrete basis functions in the Schwinger principle we have seen that the selection of such a basis set to describe the scattering of electrons by strongly polar molecules can be very difficult (Watson et al 1981). In such cases, and in others where highly accurate results may be required, it becomes necessary to use the iterative method which employs the Schwinger variational principle to solve the Lippmann-Schwinger integral equation directly (Lucchese et al 1980). The iterative Schwinger method will converge to the exact scattering matrices for the given potential provided the initial set of basis functions is adequate. However, each step in the iterative procedure is more time consuming than the initial stage of the calculation in which only discrete basis functions are used. Therefore it is desirable to develop a related variational functional which would yield improved results over those of the Schwinger principle at the non-iterative level and which would generally reduce the need for additional steps in any related iterative procedure. An attractive feature of such a method would be that it would also be implemented without any significant increase in computational effort (Watson et al 1980, Lucchese and McKoy 1979, 1980).

In an earlier paper (Takatsuka and McKoy 1981a) we proposed a new variational functional which is a straightforward modification of the Schwinger functional. There we suggested that, for a given trial scattering wavefunction, the new functional should

$\uparrow$ Permanent address: Departamento de Quimica, Universidade Federal de São Carlos, São Carlos, Brasil. $\ddagger$ Contribution no 6457 . 
give a more accurate $K$ matrix than the original Schwinger principle at the uniterated level. We also stressed that the implementation of this new functional would require only minor modifications of the computer programs which are currently used for calculations with the Schwinger principle (Lucchese et al 1980). The main purpose of this paper is to verify the above statements both mathematically and numerically.

In $\S 2$ we will prove that the $K$ matrix resulting from our new variational functional should be as accurate as that obtained from the first iteration in the iterative Schwinger method. This result is encouraging since we have already shown that the Schwinger principle gives a $K$ matrix one rank higher than the Kohn principle (Takatsuka and McKoy 1980, Takatsuka et al 1981). Section 2 presents the results of some applications of this new functional to the scattering of electrons by $\mathrm{LiH}, \mathrm{CO}^{+}, \mathrm{H}_{2}$ and $\mathrm{H}_{2}^{+}$. The results are encouraging and support our mathematical relationship between this new functional and the Schwinger variational principle. We also discuss an iterative method based on our new functional which can be expected to converge to the correct solution with fewer iterations.

\section{Theory}

The essential derivation of our new variational functional for the $K$ matrix has been described in an earlier paper (Takatsuka and McKoy 1981a). Here we will discuss some features of the method which are of importance in the present application.

Within the fixed-nuclei formulation of electron-molecule scattering, the scattering function $\psi_{k}(r)$ satisfies (in atomic units)

$$
\left(\frac{1}{2} k^{2}-H_{0}(\boldsymbol{r})-V(\boldsymbol{R}, \boldsymbol{r})\right) \psi_{k}=0
$$

where $\boldsymbol{k}$ is the incident wavevector and the effective potential $V(\boldsymbol{R}, \boldsymbol{r})$ depends parametrically on the internuclear distance, $\boldsymbol{R}$. The unperturbed Hamiltonian, $H_{0}$, is the kinetic energy operator and, in the case of ion scattering, also includes the Coulomb potential. The partial-wave components of $\psi_{k}$ are defined by

$$
\psi_{k}=\sum_{l, m} \mathrm{i}^{l} \psi_{k l m}(\boldsymbol{r}) Y_{l m}^{*}(\hat{k})
$$

and independently satisfy the same Schrödinger equation as $\psi_{k}$ itself, i.e.

$$
\left(\frac{1}{2} k^{2}-H_{0}(\boldsymbol{r})-V(\boldsymbol{R}, \boldsymbol{r})\right) \psi_{k l m}(\boldsymbol{r})=0
$$

for all $l, m$.

The Schwinger variational principle is applied direct to equation (3) without any further reduction to coupled partial-wave equationst. Using the Green's function for $H_{0}$ the Lippmann-Schwinger equation is

$$
\psi_{k l m}=S_{k l m}+G_{0} V \psi_{k l m}
$$

where $S_{k l m}$ is the incident partial wave defined similarly to equation (2). The Schwinger

$\dagger$ This point can be important in comparing the results of Schwinger variational calculations and those of numerical solutions of the coupled equations. As pointed out previously (Takatsuka and McKoy 1981b), the results of the Schwinger variational principle can be better than the exact values from the solution of the coupled equations if the Green's function in the variational expression is not restricted to the same partial-wave expansions which define the coupled equation. For example, the Green's function in equation (4) could be expanded to a higher order in spherical harmonics than is used in equation (3) to determine the coupled equation. See the results of Lucchese et al (1980) and Collins et al (1980). 
variational functional for the $K$ matrix is

$$
-\frac{1}{2}\left[K_{i j}\right]=\frac{\left\langle\psi_{i}|V| S_{j}\right\rangle\left\langle S_{i}|V| \psi_{j}\right\rangle}{\left\langle\psi_{i}\left|V-V G_{0} V\right| \psi_{j}\right\rangle}
$$

where $G_{0}$ is the standing-wave Green's function and the index $i$ specifies the values of $l$ and $m$ collectively. As usual, the stationary condition for $K_{i j}$ with respect to the expansion coefficient of $\psi_{i}$ which is

$$
\left|\psi_{i}\right\rangle=\sum_{\alpha} a_{i \alpha}|\alpha\rangle
$$

gives

$$
-\frac{1}{2} K_{i j}=\sum_{\alpha, \beta}\left\langle S_{i}|V| \alpha\right\rangle\left(D^{-1}\right)_{\alpha \beta}\left\langle\beta|V| S_{j}\right\rangle
$$

where

$$
D_{\alpha \beta}=\left\langle\alpha\left|V-V G_{0} V\right| \beta\right\rangle .
$$

The numerical evaluation of the matrix elements has been discussed by Fliflet and McKoy (1978) and Lucchese and McKoy (1980).

Our new variational principle is based on the use of another integral equation instead of equation (4), i.e.

$$
\tilde{C}_{i}=G_{0} V S_{i}+G_{0} V \tilde{C}_{i}
$$

where

$$
\tilde{C}_{i}=\psi_{i}-S_{i}
$$

The variational functional associated with equation (9) is

$$
\left[X_{i j}\right]=\frac{\left\langle\tilde{C}_{i}\left|V G_{0} V\right| S_{j}\right\rangle\left\langle S_{i}\left|V G_{0} V\right| \tilde{C}_{j}\right\rangle}{\left\langle\tilde{C}_{i}\left|V-V G_{0} V\right| \tilde{C}_{j}\right\rangle} .
$$

We will refer to this functional as the $\tilde{C}$ functional. This functional is stationary around the exact $\tilde{C}_{i}$ with a stationary value given by

$$
X_{i j}=\sum_{\alpha, \beta}\left\langle S_{i}\left|V G_{0} V\right| \alpha\right\rangle\left(D^{-1}\right)_{\alpha \beta}\left\langle\beta\left|V G_{0} V\right| S_{j}\right\rangle
$$

where the matrix $D$ is also defined by equation (8). The $K$ matrix is then given in terms of the first and second Born terms and $X_{i j}$

$$
-\frac{1}{2} K_{i j}=\left\langle S_{i}|V| S_{j}\right\rangle+\left\langle S_{i}\left|V G_{0} V\right| S_{j}\right\rangle+X_{i j}
$$

Before assessing the possible merits of equation (13) we will note some obvious features. First, the computational effort required by equation (13) is essentially the same as that of equation (5). The numerator of $\left[X_{i j}\right]$ and the second Born term in equation (13) can be evaluated by the same algorithm used for the $V G_{0} V$ term in $D_{\alpha \beta}$ since these integrations are done numerically. Secondly, the free wave components $S_{i}$ are completely factored out from the basis set used in $\left[X_{i j}\right]$ and included exactly in the first and second Born terms. Accordingly the basis functions in the expansion of $\tilde{C}$ need only include short-range (or $L^{2}$ ) functions and possibly the regularised Neumann-type functions. In actual computations, which will be discussed later, we have used only $L^{2}$ functions and obtained encouraging results. In applications we have seen that it can be important to exclude these Neumann-type functions in trial scattering functions for 
long-range potentials such as dipole potentials since, in such cases, these short- and long-range functions can often interfere and reduce the convergence in some cases.

The essential difference between the $\tilde{C}$ functional and the original Schwinger functional is clearly in the numerators where the potential $V$ has been replaced by $V G_{0} V$. We will now examine the effect of this replacement of $V$ by $V G_{0} V$. Formally, the stationary expression for the $K$ matrix in the Schwinger principle can be written as

$$
-\frac{1}{2} K_{i j}=\left\langle S_{i}\left|V\left(V-V G_{0} V\right)^{-1} V\right| S_{j}\right\rangle .
$$

Hence, using a primitive solution of

$$
\left(V-V G_{0} V\right) \tilde{\psi}_{j}^{(0)}=V S_{i}
$$

the $K$ matrix of the Schwinger functional, $K_{i j}^{\mathrm{S}}$, can be written as

$$
-\frac{1}{2} K_{i j}^{\mathrm{S}}=\left\langle S_{i}|V| \tilde{\psi}_{j}^{(0)}\right\rangle .
$$

On the other hand, the stationary $K$ matrix given by the $\tilde{C}$ functional, $K^{C}$, can be written formally as

$-\frac{1}{2} K_{i j}^{C}=\left\langle S_{i}|V| S_{j}\right\rangle+\left\langle S_{i}\left|V G_{0} V\right| S_{j}\right\rangle+\left\langle S_{i}\left|V G_{0} V\left(V-V G_{0} V\right)^{-1} V G_{0} V\right| S_{j}\right\rangle$

which is based implicitly on the primitive solution of

$$
\left(V-V G_{0} V\right) \dot{C}_{j}^{(0)}=V G_{0} V S_{j} .
$$

Next, we consider the successive iterations of the integral equation for $\tilde{C}_{j}$, equation (9), i.e.

$$
\tilde{C}_{j}^{(1)}=G_{0} V S_{j}+G_{0} V \tilde{C}_{j}^{(0)} .
$$

Defining $\tilde{\psi}_{i}^{(1)}$ as the solution of one rank higher than $\tilde{\psi}_{j}^{(0)}$ of equation (15) we have

$$
G_{0} V \tilde{C}_{j}^{(0)}=\tilde{\psi}_{j}^{(1)}-S_{j}-G_{0} V S_{j}
$$

since

$$
\tilde{\psi}_{j}^{(1)}=S_{j}+\tilde{C}_{j}^{(1)} .
$$

Next, we use equations (20) and (19) in equation (18) to obtain

$$
-\frac{1}{2} K_{i j}^{C}=\left\langle S_{i}|V| \tilde{\psi}_{j}^{(1)}\right\rangle .
$$

A comparison of equations (16) and (22) shows that the $K$ matrix obtained from the $\tilde{C}$ functional is one rank higher than the $K$ matrix of the Schwinger variational principle. We therefore expect $K^{C}$ to be more accurate than $K^{\mathrm{S}}$ and furthermore $K^{C}$ should be roughly comparable to the $K$ matrix obtained after one iteration of the iterative Schwinger method. Moreover, Watson et al (1981) and Lucchese et al (1980) have also observed that the first iteration of the Schwinger iterative method can sometimes drastically improve the non-iterative Schwinger $K$ matrix leading to a result much closer to the exact value. Thus, we can expect that the $\dot{C}$ functional should generally yield reliable $K$ matrices.

Finally, we mention that an iterative method (Lucchese et al 1980) can also be developed based on the $\tilde{C}$ functional. Although the iterative procedure can be time consuming, it is very helpful in selecting an adequate basis set for calculations at the 
non-iterative level and need only be applied to a few energies to provide some reliable reference values. Some examples of the iterative $\tilde{C}$ functional will be given in the next section where we will see that usually one iteration can yield quite accurate results.

\section{Results and discussion}

In this section we present results of some applications of the $\tilde{C}$-functional method to the scattering of electrons by $\mathrm{H}_{2}, \mathrm{H}_{2}^{+}, \mathrm{CO}^{+}$and $\mathrm{LiH}$. These applications were primarily chosen to assess the relationship which we established above between the iterative Schwinger method and the $\tilde{C}$-functional method and do not represent an extensive numerical study of the $\tilde{C}$ functional itself. For example, we did not try to obtain optimum basis set expansions for the $\tilde{C}$-functional method itself but simply chose basis set expansions which had been used in previous calculations with the iterative Schwinger method and applied these in the $\tilde{C}$-functional method. All calculations were carried out in the body-fixed frame and within the static-exchange approximation.

Our first applications are to the scattering of electrons by the simple system $\mathrm{H}_{2}$ and $\mathrm{H}_{2}^{+}$. For $\mathrm{H}_{2}$ the SCF wavefunction is obtained at an internuclear distance of $1.4006 a_{0}$ with the $5 \mathrm{~s} 2 z$ Cartesian Gaussian basis set used previously by Watson et al (1980). The scattering basis contains two $\mathrm{s}$ and two $z$ Cartesian Gaussian functions both with exponents of 1.0 and 0.3 . In table 1 we compare the ${ }^{2} \Sigma_{\mathrm{g}} K$-matrix elements and eigenphase sums for $\mathrm{e}-\mathrm{H}_{2}$ scattering at momenta of 0.1 and 0.5 obtained by the

Table 1. $K$-matrix elements and eigenphase sums for e- $\mathrm{H}_{2}\left({ }^{2} \Sigma_{\mathrm{g}}\right)$ and $\mathrm{e}-\mathrm{H}_{2}^{+}\left({ }^{1} \Sigma_{\mathrm{u}}\right)$ scattering.

\begin{tabular}{|c|c|c|c|c|}
\hline \multirow[b]{2}{*}{ Iteration } & \multicolumn{3}{|c|}{ Iterative Schwinger method } & \multirow{2}{*}{$\begin{array}{l}\tilde{C} \text { functional } \\
0\end{array}$} \\
\hline & 0 & 1 & 2 & \\
\hline \multicolumn{5}{|c|}{$\mathrm{e}-\mathrm{H}_{2}\left({ }^{2} \Sigma_{\mathrm{g}}\right), k=0.1^{\mathrm{a}}$} \\
\hline$K_{00}$ & $-0.278(0)^{\mathrm{b}}$ & $-0.210(0)$ & $-0.216(0)$ & $-0.256(0)$ \\
\hline$K_{02}$ & $0.312(-4)$ & $0.405(-2)$ & $0.405(-2)$ & $0.401(-2)$ \\
\hline$K_{22}$ & $0.175(-5)$ & $0.165(-2)$ & $0.165(-2)$ & $0.165(-2)$ \\
\hline$K_{24}$ & $<0.1(-9)$ & $0.390(-3)$ & $0.390(-3)$ & $0.390(-3)$ \\
\hline$K_{44}$ & $<0.1(-9)$ & $0.642(-4)$ & $0.642(-4)$ & $0.642(-4)$ \\
\hline$\delta_{\text {SUM }}{ }^{c}$ & $2.870(0)$ & $2.935(0)$ & $2.935(0)$ & $2.892(0)$ \\
\hline \multicolumn{5}{|c|}{$\mathrm{e}-\mathrm{H}_{2}\left({ }^{2} \Sigma_{\mathrm{g}}\right), k=0.5^{\mathrm{a}}$} \\
\hline$K_{00}$ & $-2.079(0)$ & $-1.545(0)$ & $-1.545(0)$ & $-1.679(0)$ \\
\hline$K_{02}$ & $0.435(-3)$ & $0.132(-1)$ & $0.132(-1)$ & $0.162(-1)$ \\
\hline$K_{22}$ & $0.427(-2)$ & $0.164(-1)$ & $0.164(-1)$ & $0.163(-1)$ \\
\hline$K_{24}$ & $0.485(-4)$ & $0.258(-2)$ & $0.258(-2)$ & $0.258(-2)$ \\
\hline$K_{44}$ & $0.575(-6)$ & $0.288(-2)$ & $0.288(-2)$ & $0.288(-2)$ \\
\hline$\delta_{\text {SUM }}$ & $2.023(0)$ & $2.166(0)$ & $2.166(0)$ & $2.131(0)$ \\
\hline \multicolumn{5}{|c|}{$\mathrm{e}-\mathrm{H}_{2}^{+}\left({ }^{1} \Sigma_{\mathrm{u}}\right), k=0.6062^{\mathrm{a}}$} \\
\hline$K_{11}$ & $0.241(0)$ & $0.257(0)$ & $0.257(0)$ & $0.255(0)$ \\
\hline$K_{13}$ & $0.205(-1)$ & $0.562(-2)$ & $0.562(-2)$ & $0.562(-2)$ \\
\hline$K_{33}$ & $0.739(-2)$ & $0.161(-1)$ & $0.161(-1)$ & $0.161(-1)$ \\
\hline$\delta_{\text {SUM }}$ & $0.244(0)$ & $0.276(0)$ & $0.276(0)$ & $0.276(0)$ \\
\hline
\end{tabular}

${ }^{a}$ Incident momentum.

${ }^{b}$ The numbers in parentheses indicate multiplicative power of 10 .

${ }^{c}$ Eigenphase sum in radians. 
$\tilde{C}$-functional method and the iterative Schwinger method (Lucchese et al 1980). These results show that the $K$-matrix elements, given by the $\tilde{C}$-functional method with these four discrete basis functions without any iterations, that is, for $n=0$ in table 1 , are quite close to the $K$-matrix elements after one iteration in the iterative Schwinger method. This trend agrees well with the mathematical relationship which we have established between these two variational functionals. For this simple system the results almost converge after one iteration.

In table 1 we also show results for the scattering of electrons by $\mathrm{H}_{2}^{+}$for an incident momentum of $0.6062 \mathrm{au}$ in the ${ }^{1} \Sigma_{\mathrm{u}}$ channel. In these calculations we use the $1 \sigma_{\mathrm{g}}$ molecular orbital of $\mathrm{H}_{2}$ at $R=1.4006 a_{0}$ as the target orbital. Again the agreement between the $\tilde{C}$-functional results without any iterations and those of the iterative Schwinger method after one iteration is good.

Next we consider the scattering of electrons by $\mathrm{CO}^{+}\left(\mathrm{X}^{2} \Sigma^{+}\right)$, a molecular ion with a significant dipole moment. The static-exchange potential of this ion is obtained from the CO Hartree-Fock wavefunction of McLean and Yoshimine (1967) at an internuclear separation of of $2.132 a_{0}$. With this frozen-core wavefunction the dipole and quadrupole moments are found to be 1.828 and $-2.06 \mathrm{au}$, respectively. For the scattering calculation we choose the sixteen basis functions shown in table 2 . In table 3 we show the $K$ matrices and eigenphase sums obtained using the basis set of table 2 in the $\tilde{C}$ functional and in the Schwinger variational principle, that is, the $n=0$ column of table 3 . In the same table we also show the $K$ matrices resulting from three steps in the iterative Schwinger method. These $K$ matrices are calculated with respect to a coordinate system at the centre of mass of the molecule.

A comparison of the results of table 3 shows that the $K$-matrix elements of the $\tilde{C}$-functional method are generally as good or better than those given after one iteration in the iterative Schwinger method. For those matrix elements, $K_{l l}$, with low $l$ values the correspondence between the $\tilde{C}$-functional results and those from one iteration in the iterative Schwinger method is quite evident; for example, the elements $K_{00}, K_{01}$ and $K_{02}$. For those elements, $K_{l l}$, with higher $l$ and $l^{\prime}$ values the $\tilde{C}$ functional gives results which are closer to those of a larger number of iterations, for example, $K_{12}$. The

Table 2. Basis set used on the e-CO${ }^{+}\left({ }^{1} \Sigma\right)$ scattering ${ }^{\mathrm{a}}$.

\begin{tabular}{llccll}
\hline$(l, m, m)$ & Centre & Exponent & $(l, m, n)$ & Centre & Exponent \\
\hline$(0,0,0)$ & $\mathrm{C}$ & 10.0 & $(0,0,0)$ & $\mathrm{CM}^{\mathrm{b}}$ & 7.0 \\
$(0,0,0)$ & $\mathrm{C}$ & 1.0 & $(0,0,0)$ & $\mathrm{CM}$ & 1.0 \\
$(0,0,0)$ & $\mathrm{C}$ & 0.1 & $(0,0,0)$ & $\mathrm{CM}$ & 0.1 \\
$(0,0,1)$ & $\mathrm{C}$ & 0.5 & $(0,0,1)$ & $\mathrm{CM}$ & 0.5 \\
$(0,0,1)$ & $\mathrm{C}$ & 0.2 & $(0,0,1)$ & $\mathrm{CM}$ & 0.1 \\
$(0,0,0)$ & $\mathrm{O}$ & 4.0 & $(0,0,2)$ & $\mathrm{CM}$ & 0.1 \\
$(0,0,0)$ & $\mathrm{O}$ & 0.5 & & & \\
$(0,0,0)$ & $\mathrm{O}$ & 0.1 & & & \\
$(0,0,1)$ & $\mathrm{O}$ & 0.5 & & & \\
$(0,0,1)$ & $\mathrm{O}$ & 0.2 & & & \\
\hline
\end{tabular}

${ }^{a}$ Cartesian Gaussian function of the form

$$
\mu_{l m n}=N\left(x-A_{x}\right)^{l}\left(y-A_{y}\right)^{m}\left(z-A_{z}\right)^{n} \exp \left(-\alpha|r-\boldsymbol{A}|^{2}\right)
$$

${ }^{\mathrm{b}}$ Centre of mass. 
Table 3. $K$-matrix elements and eigenphase sums for $\mathrm{e}-\mathrm{CO}^{+}\left({ }^{1} \Sigma\right)$ scattering at $k=$ $0.8573^{\mathrm{b}}$.

\begin{tabular}{|c|c|c|c|c|c|}
\hline \multirow[b]{2}{*}{ Iteration } & \multicolumn{4}{|c|}{ Iterative Schwinger method } & \multirow{2}{*}{$\begin{array}{l}\tilde{C} \text { functional } \\
0\end{array}$} \\
\hline & 0 & 1 & 2 & 3 & \\
\hline$K_{00}$ & -0.384 & -0.694 & -0.846 & -0.842 & -0.639 \\
\hline$K_{01}$ & 0.981 & 0.472 & 0.123 & 0.132 & 0.407 \\
\hline$K_{02}$ & 1.606 & 1.853 & 1.585 & 1.594 & 1.891 \\
\hline$K_{11}$ & 1.915 & 2.493 & 1.687 & 1.700 & 1.747 \\
\hline$K_{12}$ & 0.170 & 1.667 & 1.049 & 1.067 & 0.915 \\
\hline$K_{22}$ & -0.804 & 0.256 & -0.215 & -0.197 & -0.358 \\
\hline$\delta_{\text {SUM }}{ }^{\mathrm{c}}$ & 0.882 & 1.011 & 0.772 & 0.773 & 0.846 \\
\hline
\end{tabular}

${ }^{a}$ With the coordinate system at the centre of mass.

${ }^{b}$ Incident momentum.

${ }^{c}$ Eigenphase sum in radians.

explanation of this behaviour is probably that for these elements the centrifugal barrier causes the regular solution, $S_{l}$, to dominate the scattering function, $\tilde{\psi}_{l}$. Since the $\tilde{C}$ functional determines this difference, $\tilde{\psi}_{l}-S_{l}$, the results of the method can be expected to be quite accurate for these cases. We emphasise that we did not try to optimise the basis set used in these $\tilde{C}$-functional calculations. In actual applications one would optimise the basis set for the $\tilde{C}$ functional at some energy and then use this basis to solve the scattering problem at many other energies.

The results in table 3 also show clearly that the eigenphase sums can often be a very poor measure of the quality or convergence of a given calculation. This is particularly important for long-range potentials. For example, the Schwinger variational method, that is, $n=0$ of table 3 , gives an eigenphase sum of $0.882 \mathrm{rad}$ which is quite close to the converged value of 0.773 , even though the corresponding $K$-matrix elements are clearly very different. We believe that this behaviour also occurs with other methods of solving the collision equation and hence it is important to present the actual $K$-matrix elements along with the eigenphase sums.

Recently Watson et al (1981) applied the iterative Schwinger method to study e- $\mathrm{LiH}$ collisions. Hence, as a final example, we now use the $\tilde{C}$ functional to study the scattering of electrons by this strongly polar molecule. In these studies we use the same SCF wavefunction as Watson et al (1981). At an internuclear distance of $3.015 a_{0}$ this wavefunction gives a dipole moment of $2.369 \mathrm{au}$. We also use the same scattering basis sets of thirteen and seven Cartesian Gaussian functions for the ${ }^{2} \Sigma$ and ${ }^{2} \Pi$ symmetrics, respectively (Watson et al 1981).

In table 4 we compare the $K$-matrix elements obtained by the $\tilde{C}$-functional method and the iterative Schwinger method at $k=0.5$ au for the ${ }^{2} \Sigma$ channel and $k=0.1$ au for the ${ }^{2} \Pi$ channel. We note again the approximate equivalence between the results of the $\tilde{C}$-functional method with discrete basis functions only and those of the iterative Schwinger method after one iteration. The basis set used on these calculations was not optimised for the $\tilde{C}$-functional method but was simply chosen to be the same as the basis used previously in the iterative Schwinger method (Watson et al 1981). Optimisation of this basis set or choosing a larger basis in the $\tilde{C}$ functional should give even more accurate results still without the need for any iterations. For a further comparison 
Table 4. $K$-matrix elements and eigenphase sums for e- $\mathrm{LiH}$ scattering ${ }^{\mathrm{a}}$.

\begin{tabular}{|c|c|c|c|c|c|}
\hline \multirow[b]{2}{*}{ Iteration } & \multicolumn{3}{|c|}{ Iterative Schwinger method } & \multicolumn{2}{|c|}{$\check{C}$ functional } \\
\hline & 0 & 1 & 2 & 0 & 1 \\
\hline \multicolumn{6}{|c|}{$k=0.5\left({ }^{2} \Sigma\right)^{\mathrm{b}}$} \\
\hline$K_{00}$ & 0.268 & 0.916 & 0.921 & 0.904 & 0.922 \\
\hline$K_{01}$ & -1.475 & -0.304 & -0.233 & -0.346 & -0.215 \\
\hline$K_{02}$ & -0.136 & 0.282 & 0.316 & 0.180 & 0.328 \\
\hline$K_{11}$ & -3.075 & -4.227 & -4.280 & -3.237 & -4.120 \\
\hline$K_{12}$ & -1.185 & -2.392 & -2.614 & -2.036 & -2.526 \\
\hline$K_{22}$ & -0.258 & -0.862 & -0.967 & -0.773 & -0.899 \\
\hline$K_{13}$ & -0.025 & -0.724 & -0.781 & -0.294 & -0.736 \\
\hline$K_{23}$ & -0.028 & -0.722 & -0.817 & -0.573 & -0.793 \\
\hline$K_{33}$ & -0.008 & -0.177 & -0.211 & -0.094 & -0.202 \\
\hline$\delta_{\text {SUM }}{ }^{c}$ & 2.558 & 2.698 & 2.751 & 2.733 & 2.769 \\
\hline \multicolumn{6}{|c|}{$k=0.1\left({ }^{2} \Pi\right)$} \\
\hline$K_{11}$ & 0.009 & 0.419 & 0.750 & 0.493 & 0.788 \\
\hline$K_{12}$ & -0.001 & -0.449 & -0.544 & -0.504 & -0.500 \\
\hline$K_{22}$ & $\simeq 0.2 \times 10^{-4}$ & -0.050 & -0.053 & -0.060 & -0.051 \\
\hline$K_{13}$ & $\approx 0.1 \times 10^{-4}$ & 0.012 & 0.071 & 0.017 & 0.090 \\
\hline$K_{23}$ & $\simeq 0.2 \times 10^{-5}$ & -0.361 & -0.417 & -0.364 & -0.402 \\
\hline$K_{33}$ & $\simeq 0.0$ & -0.023 & -0.020 & -0.022 & -0.011 \\
\hline$\delta_{\mathrm{SUM}}$ & 0.009 & 0.269 & 0.454 & 0.304 & 0.493 \\
\hline
\end{tabular}

${ }^{a}$ With the coordinate system at the centre of mass.

${ }^{\mathrm{b}}$ Incident momentum.

${ }^{c}$ Eigenphase sum in radians.

we also show the results after one iteration in the $\tilde{C}$-functional method. The iterative procedure for the $\tilde{C}$ functional is implemented in a similar way to that of the Schwinger method. In fact, the procedure requires very slight changes in the computer programs used in the Schwinger method.

\section{Conclusions}

We have discussed various features of our recently proposed variational functional for scattering theory ${ }^{\dagger}$ (Takatsuka and McKoy 1981a). We have shown that this new functional can provide results with a purely discrete set of functions which are equivalent to those obtained from the first iteration in the iterative Schwinger method (Lucchese et al 1980). Applications of this new functional to the scattering of electrons by several systems including $\mathrm{CO}^{+}$and $\mathrm{LiH}$ illustrate this relationship and other features of the method. The results of these applications suggest that this functional can provide quite accurate results directly with a set of discrete basis functions. This feature also suggests that this functional can be very effective in the study of collisions of low-energy

+ After the completion of the work presented in this paper, it was brought to our attention that this variational functional has been previously discussed (Kolsrud 1958). However, the method was only applied to an attractive exponential potential and no discussion of the relation between it and other variational expressions was presented. 
electrons with non-linear polyatomic molecules both at the single channel and multichannel levels. This aspect of our work including polarisation effects will be presented elsewhere (Takatsuka and McKoy 1981c).

\section{Acknowledgments}

We would like to thank Robert Lucchese for many useful discussions and assistance during the computational course of this work. One of us (LMT) acknowledges the financial support of FAPESP (São Paulo, Brasil). This research is based upon work supported by the National Science Foundation under grant No CHE79-15807. The research reported in this paper made use of the Dreyfus-NSF Theoretical Chemistry Computer which was funded through grants from the Camille and Henry Dreyfus Foundation, the National Science Foundation (grant No CHE78-20235) and the Sloan Fund of the California Institute of Technology. This research was also supported in part by an Institutional Grant from the United States Department of Energy, No EY-76-G03-1305.

\section{References}

Blatt J M and Jackson J D 1949 Phys. Rev. 26 18-37

Collins L A, Robb W D and Morrison M A 1980 Phys. Rev. A 21 488-95

Fliflet A W and McKoy V 1978 Phys. Rev. A $181048-54$

Kato T 1951 Prog. Theor. Phys. 6 295-305

Kolsrud M 1958 Phys. Rev. 112 1436-7

Lucchese R R and McKoy V 1979 J. Phys. B: At. Mol. Phys. 12 L421-4

- 1980 Phys. Rev. A 21 112-23

Lucchese R R, Watson D K and McKoy V 1980 Phys. Rev. A 22 421-6

McLean A D and Yoshimine M 1967 Tables of Linear Molecule Wave Functions (San Jose, California: IBM San Jose Research Laboratory) p 27

Takatsuka K, Lucchese R R and McKoy V 1981 Phys. Rev. A accepted for publication

Takatsuka K and McKoy V 1980 Phys. Rev. Lett. $451396-9$

- 1981a Phys. Rev. A 23 2352-8

- 1981b Phys. Rev. A accepted for publication

- 1981c in preparation

Watson D K, Lucchese R R, McKoy V and Rescigno T N 1980 Phys. Rev. A $21738-44$

Watson D K, Rescigno T N and McKoy V 1981 J. Phys. B: At. Mol. Phys. 14 1875-82 